\title{
In situ Surface Chemistry Engineering of Cobalt- Sulfide Nanosheets for Improved Oxygen Evolution Activity
}

Suxiao Ju, ${ }^{\dagger}$ Yuanjun Liu,,${ }^{* \dagger}$ Hui Chen, ${ }^{\dagger}$ Fujiang Tan, ${ }^{\dagger}$ Aihua Yuan,,${ }^{*}{ }^{\dagger}$ Xiaoyun Li, ${ }^{\dagger}$ and Guoxing $\mathrm{Zhu}^{*} \stackrel{+}{\ddagger}$

${ }^{\dagger}$ School of Environmental and Chemical Engineering, Jiangsu University of Science and Technology, Zhenjiang 202018, China, *Email: liuyuanjun@just.edu.cn; aihua.yuan@just.edu.cn

'School of Chemistry and Chemical Engineering, Jiangsu University, Zhenjiang 212013, China, *E-mail: zhuguoxing@ujs.edu.cn 

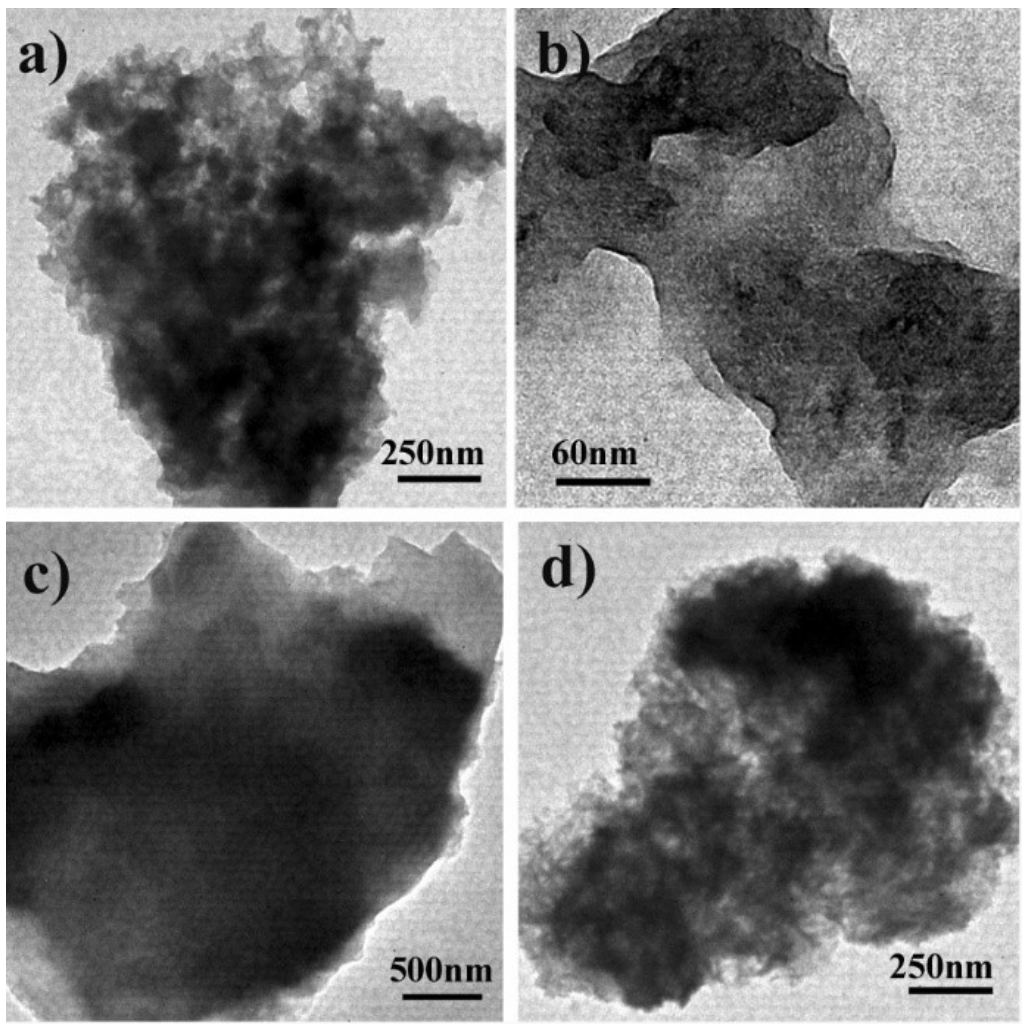

Figure S1. TEM images of various cobalt sulfide products. a) Co-S-16, b) Co-S-60, c) Co-S-150, d) Co-S-190.

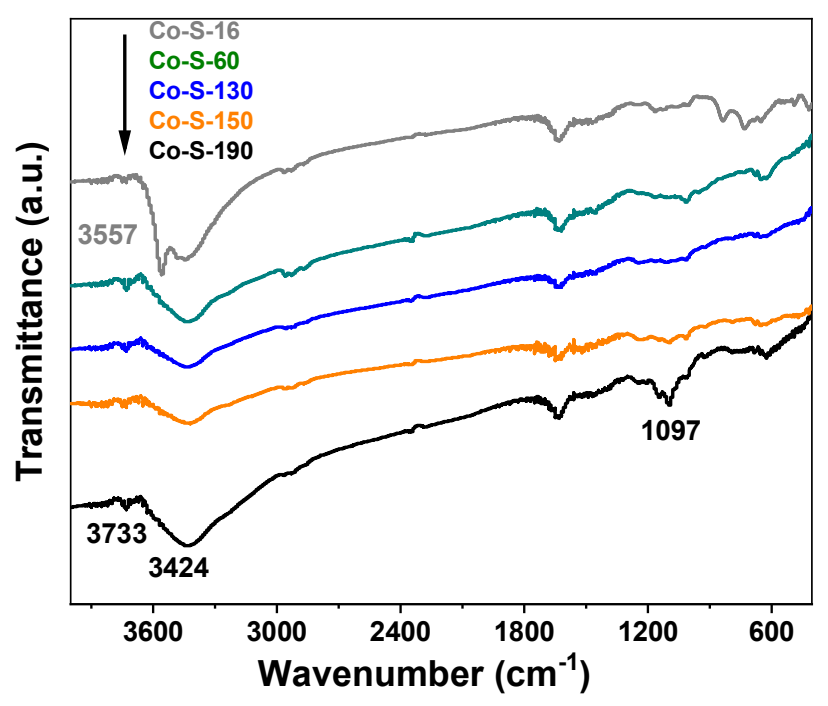

Figure S2. IR spectra of the obtained cobalt sulfide products. 


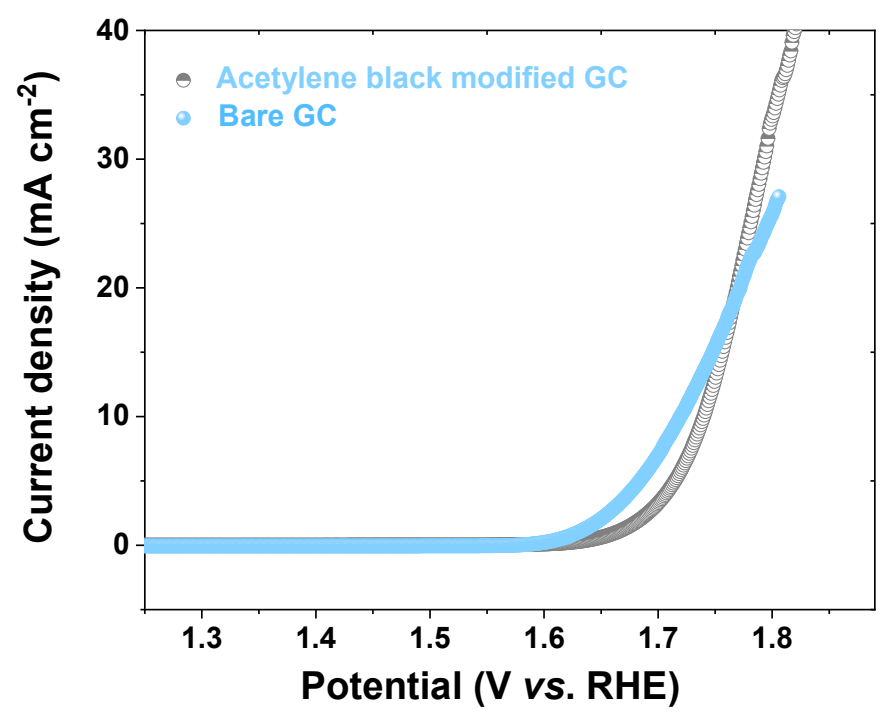

Figure S3. LSV curves of the bare glassy carbon electrode (GC) and GC modified by acetylene black.
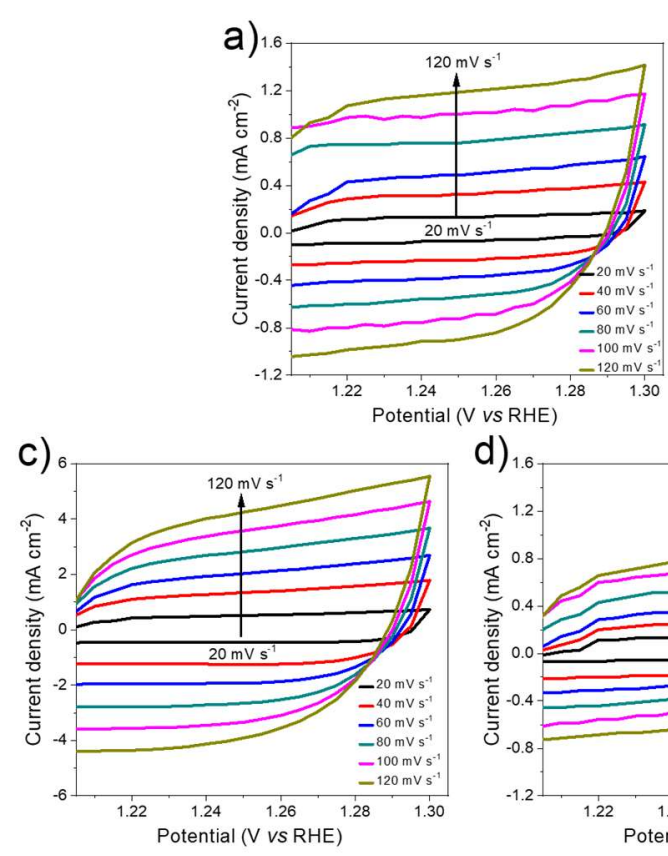

d)

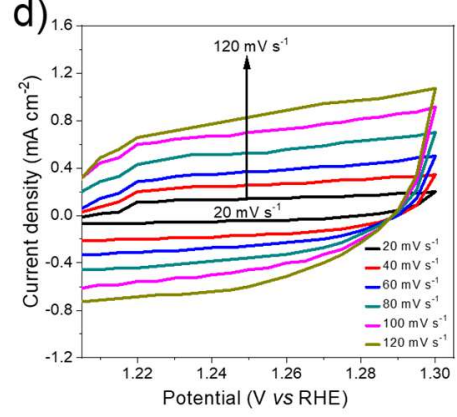

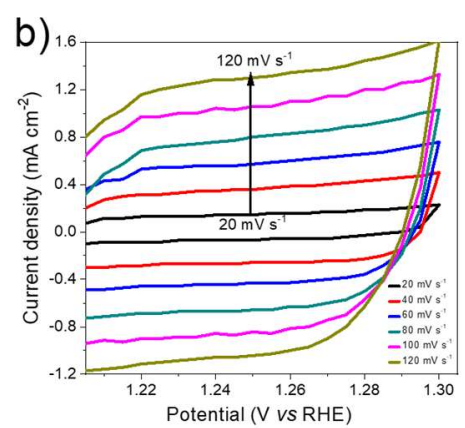

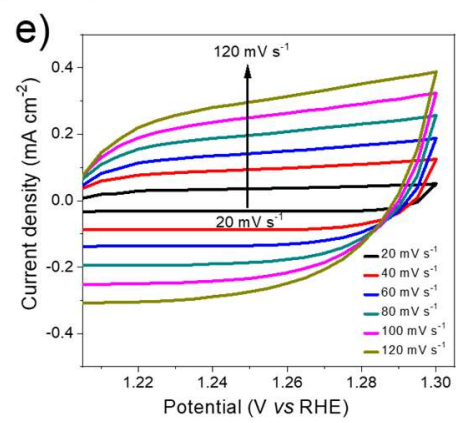

Figure S4. Cyclic voltammetry curves of a) Co-S-190, b) Co-S-150, c) Co-S-130, d) Co-S-60, e) Co-S-16 products with different scanning rates. 

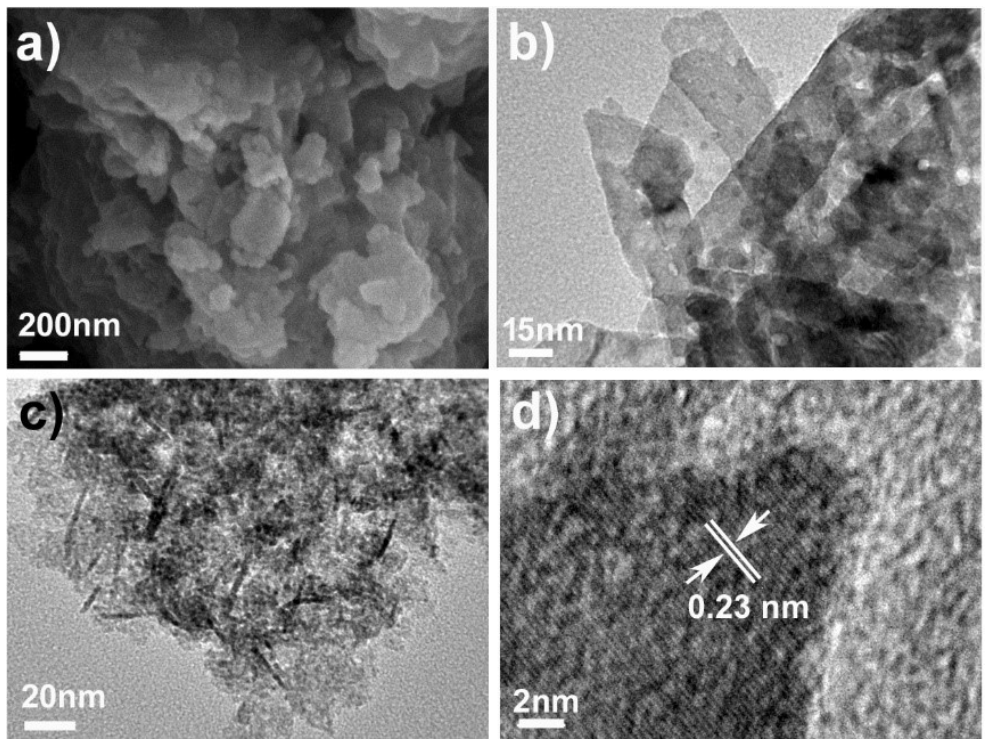

Figure S5. a) SEM, b, c) TEM, and d) HRTEM images of the Co-S-130 product after $36 \mathrm{~h}$ of OER testing.
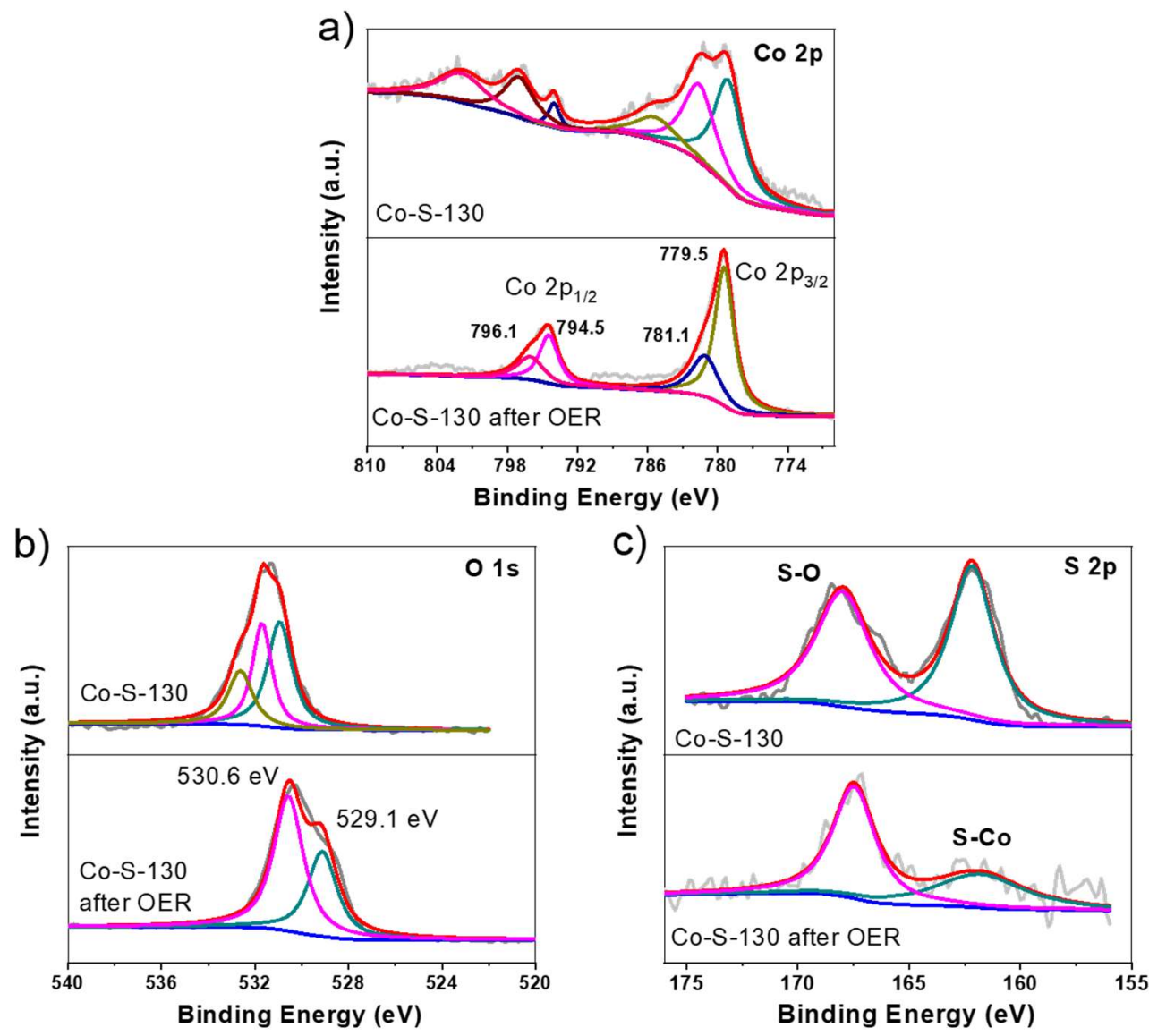

Figure S6. XPS spectra of a) cobalt, b) oxygen, and c) sulfur of the Co-S-130 catalyst after $36 \mathrm{~h}$ of OER testing. For comparison, the XPS spectra of original Co-S-130 catalyst were also shown. 
Table S1. The composition of the obtained cobalt sulfide products analysed by XPS and ICP. ${ }^{\text {a }}$

\begin{tabular}{llc}
\hline Samples & $\begin{array}{l}\mathrm{Co}_{\mathbf{x}} \mathrm{S}_{\mathbf{y}} \mathrm{O}_{\mathbf{z}} \text { analyzed } \\
\text { by XPS }\end{array}$ & $\begin{array}{l}\text { Co content analyzed } \\
\text { by ICP (wt \%) }\end{array}$ \\
\hline Co-S-16 & $\mathrm{CoS}_{0.13} \mathrm{O}_{2.3}$ & 53.8 \\
Co-S-60 & $\mathrm{CoS}_{2.5} \mathrm{O}_{4.6}$ & 33.3 \\
Co-S-130 & $\mathrm{CoS}_{3.0} \mathrm{O}_{5.2}$ & 36.7 \\
Co-S-160 & $\mathrm{CoS}_{2.7} \mathrm{O}_{7.4}$ & 32.0 \\
Co-S-190 & $\mathrm{CoS}_{3.6} \mathrm{O}_{15.0}$ & 31.5 \\
Co-S-130 after 36 h & $\mathrm{CoS}_{0.1} \mathrm{O}_{2.7}$ & 36.4 \\
of OER & & \\
\hline
\end{tabular}

${ }^{\text {a } B e ~ n o t e d ~ t h a t ~ t h e ~ c o m p o s i t i o n ~ a n a l y s i s ~ b y ~ X P S ~ r e s u l t s ~ i s ~ s e m i-q u a n t i t a t i v e . ~}$ Especially, the oxygen contents determined by XPS are not accurate but may have reference meaning because during the sample treatment and characterization process, some oxygen-containing substances such as water, carbon dioxide will be absorbed on the material surface. In addition, the surface oxidation of sulfides that is dependent on the surface sulfur content is also inevitable, which will also introduce oxygen on the surface. In our case, there are at least three possible oxygen species in the samples: lattice oxygen, adsorbed oxygen species including water, carbon dioxide, oxygen in oxidized sulfur species. 
Table S2. Comparison of the catalytic performance of our catalysts with the reported cobalt sulfides. ${ }^{\mathrm{a}}$

\begin{tabular}{|c|c|c|c|}
\hline Catalysts & Substrates & $\begin{array}{c}\text { Overpotentials }(\mathrm{mV}) \\
\text { at } 10 \mathrm{~mA} \mathrm{~cm}^{-2}\end{array}$ & Refs. \\
\hline \multirow{2}{*}{ Cobalt sulfide $\left(\mathrm{CoS}_{2}\right)$ nanosheets } & \multirow{2}{*}{ GCE } & $338^{\mathrm{b}}$ & \multirow{2}{*}{ this work } \\
\hline & & $312^{\mathrm{c}}$ & \\
\hline CoS nanosheets on carbon cloth & Carbon cloth & 390 & 1 \\
\hline $\mathrm{Co}_{9} \mathrm{~S}_{8}$ microplates & $\mathrm{RDE}^{\mathrm{d}}$ & 278 & 2 \\
\hline $\mathrm{Co}_{9} \mathrm{~S}_{8}$ hollow nanocages & GCE & 400 & 3 \\
\hline $\mathrm{Co}_{3} \mathrm{~S}_{4}$ on carbon cloth & Carbon cloth & 360 & 4 \\
\hline $\mathrm{CoS}_{2}$ nanosheets & GCE & 339 & 5 \\
\hline $\mathrm{CoS}_{2}$ film on Ti-mesh & Ti-mesh & 361 & 6 \\
\hline Hierarchical cobalt sulfide & GCE & 275 & 7 \\
\hline $\mathrm{CoS}$ & GCE & 440 & 8 \\
\hline $\mathrm{Co}_{9} \mathrm{~S}_{8}$ & GCE & 380 & 9 \\
\hline $\mathrm{Co}_{9} \mathrm{~S}_{8}$ hollow spheres & GCE & 285 & 10 \\
\hline Mesoporous $\mathrm{CoS}_{1.097}$ nanotubes & $\mathrm{RDE}^{\mathrm{d}}$ & 331 & 11 \\
\hline Porous $\mathrm{Co}_{1-\mathrm{x}} \mathrm{S}$ microplates & GCE & 311 & 12 \\
\hline $\mathrm{Co}_{9} \mathrm{~S}_{8}$ on cobalt foam & Cobalt foam & 350 & 13 \\
\hline $\mathrm{RuO}_{2}$ & GCE & 380 & 14 \\
\hline $\mathrm{Co}_{0.5} \mathrm{Fe}_{0.5} \mathrm{~S} @ \mathrm{~N}-\mathrm{MC}$ & GCE & 410 & 15 \\
\hline Hierarchical pores $\mathrm{NiCo}_{2} \mathrm{~S}_{4}$ & GCE & 337 & 16 \\
\hline $\mathrm{CuCo}_{2} \mathrm{~S}_{4}$ & $\mathrm{RDE}^{\mathrm{d}}$ & 395 & 17 \\
\hline $\mathrm{CoS}_{\mathrm{x}} @ \mathrm{MoS}_{2}$ microcubes & GCE & 347 & 18 \\
\hline
\end{tabular}

${ }^{\mathrm{a}}$ in the electrolyte of $1 \mathrm{M} \mathrm{KOH},{ }^{\mathrm{b}}$ with catalyst mass loading of $0.17 \mathrm{mg} \mathrm{cm}^{-2},{ }^{\mathrm{c}}$ with catalyst mass loading of $0.37-0.45 \mathrm{mg} \mathrm{cm}^{-2},{ }^{\mathrm{d}}$ rotating disk electrode.

\section{References}

(1) Liu, B.; Qu, S.; Kou, Y.; Liu, Z.; Chen, X.; Wu, Y.; Han, X.; Deng, Y.; Hu, W.; Zhong, C., In Situ Electrodeposition of Cobalt Sulfide Nanosheet Arrays on Carbon Cloth as a Highly Efficient Bifunctional Electrocatalyst for Oxygen Evolution and Reduction Reactions. ACS Appl. Mater. Interf. 2018, 10, 30433-30440.

(2) Liu, H.; Ma, F.-X.; Xu, C.-Y.; Yang, L.; Du, Y.; Wang, P.-P.; Yang, S.; Zhen, L., SulfurizingInduced Hollowing of $\mathrm{Co}_{9} \mathrm{~S}_{8}$ Microplates with Nanosheet Units for Highly Efficient Water Oxidation. ACS Appl. Mater. Interf. 2017, 9, 11634-11641.

(3) Ganesan, V.; Ramasamy, P.; Kim, J., Hierarchical $\mathrm{Ni}_{3.5} \mathrm{Co}_{5.5} \mathrm{~S}_{8}$ Nanosheet-assembled Hollow Nanocages: Superior Electrocatalyst Towards Oxygen Evolution Reaction. Int. J. Hydrogen Energ. 2017, 42, 5985-5992.

(4) Zhu, M.; Zhang, Z.; Zhang, H.; Zhang, H.; Zhang, X.; Zhang, L.; Wang, S., Hydrophilic Cobalt Sulfide Nanosheets as a Bifunctional Catalyst for Oxygen and Hydrogen Evolution in Electrolysis of Alkaline Aqueous Solution. J. Colloid Interf. Sci. 2018, 509, 522-528. 
(5) Hua, Y.; Jiang, H.; Jiang, H.; Zhang, H.; Li, C., Hierarchical Porous $\mathrm{CoS}_{2}$ Microboxes for Efficient Oxygen Evolution Reaction. Electrochim. Acta 2018, 278, 219-225.

(6) Liu, T.; Liang, Y.; Liu, Q.; Sun, X.; He, Y.; Asiri, A. M., Electrodeposition of Cobalt-sulfide Nanosheets Film as an Efficient Electrocatalyst for Oxygen Evolution Reaction. Electrochem. Commun. 2015, 60, 92-96.

(7) Lin, R.; Lin, T.; Huang, J.; Huang, X.; Liu, Y., Hierarchical Cobalt Sulfide with Vertical In-plane Edge Structure for Enhanced Electrocatalytic Oxygen Evolution Reaction. Electrochim. Acta 2018, 281, 348-356.

(8) Prabakaran, K.; Lokanathan, M.; Kakade, B., Three Dimensional Flower Like Cobalt Sulfide (CoS)/Functionalized MWCNT Composite Catalyst for Efficient Oxygen Evolution Reactions. Appl. Surf. Sci. 2019, 466, 830-836.

(9) Zhang, S.; Sun, Y.; Liao, F.; Shen, Y.; Shi, H.; Shao, M., Cog $\mathrm{S}_{8}-\mathrm{CuS-FeS} \mathrm{Trimetal} \mathrm{Sulfides} \mathrm{for}$ Excellent Oxygen Evolution Reaction Electrocatalysis. Electrochim. Acta 2018, 283, 1695-1701.

(10) Feng, X.; Jiao, Q.; Liu, T.; Li, Q.; Yin, M.; Zhao, Y.; Li, H.; Feng, C.; Zhou, W., Facile Synthesis of $\mathrm{Co}_{9} \mathrm{~S}_{8}$ Hollow Spheres as a High-Performance Electrocatalyst for the Oxygen Evolution Reaction. ACS Sustain. Chem. Eng. 2018, 6, 1863-1871.

(11) Zhang, J.; Zhang, D.; Zhang, R.; Zhang, N.; Cui, C.; Zhang, J.; Jiang, B.; Yuan, B.; Wang, T.; Xie, H.; Li, Q., Facile Synthesis of Mesoporous and Thin-Walled Ni-Co Sulfide Nanotubes as Efficient Electrocatalysts for Oxygen Evolution Reaction. ACS Appl. Energy Mater. 2018, 1, 495-502.

(12) Liu, Y.; Zhang, J.; Li, Y.; Yuan, G.; Niu, X.; Zhang, X.; Wang, Q., Self-Templated Synthesis of Co1-xS Porous Hexagonal Microplates for Efficient Electrocatalytic Oxygen Evolution. ChemElectroChem 2018, 5, 1167-1172.

(13) Xiong, D.; Zhang, Q.; Thalluri, S. M.; Xu, J.; Li, W.; Fu, X.; Liu, L., One-Step Fabrication of Monolithic Electrodes Comprising $\mathrm{Co}_{9} \mathrm{~S}_{8}$ Particles Supported on Cobalt Foam for Efficient and Durable Oxygen Evolution Reaction. Chem. Eur. J. 2017, 23, 8749-8755.

(14) Yang, J.; Zhu, G. X.; Liu, Y. J.; Xia, J. X.; Ji, Z. Y.; Shen, X. P.; Wu, S. K., Fe $\mathrm{Fe}_{3} \mathrm{O}_{4}$-Decorated $\mathrm{Co}_{9} \mathrm{~S}_{8}$ Nanoparticles In Situ Grown on Reduced Graphene Oxide: A New and Efficient Electrocatalyst for Oxygen Evolution Reaction. Adv. Funct. Mater. 2016, 26, 4712-4721.

(15) Shen, M.; Ruan, C.; Chen, Y.; Jiang, C.; Ai, K.; Lu, L., Covalent Entrapment of Cobalt-Iron Sulfides in N-Doped Mesoporous Carbon: Extraordinary Bifunctional Electrocatalysts for Oxygen Reduction and Evolution Reactions. ACS Appl. Mater. Interf. 2015, 7, 1207-1218.

(16) Jiang, J.; Yan, C.; Zhao, X.; Luo, H.; Xue, Z.; Mu, T., A PEGylated Deep Eutectic Solvent for Controllable Solvothermal Synthesis of Porous $\mathrm{NiCo}_{2} \mathrm{~S}_{4}$ for Efficient Oxygen Evolution Reaction. Green Chem. 2017, 19, 3023-3031.

(17) Wiltrout, A. M.; Read, C. G.; Spencer, E. M.; Schaak, R. E., Solution Synthesis of Thiospinel $\mathrm{CuCo}_{2} \mathrm{~S}_{4}$ Nanoparticles. Inorg. Chem. 2016, 55, 221-226.

(18) Yang, L.; Zhang, L.; Xu, G.; Ma, X.; Wang, W.; Song, H.; Jia, D., Metal-Organic-FrameworkDerived Hollow $\mathrm{CoS}_{\mathrm{x}} @ \mathrm{MoS}_{2}$ Microcubes as Superior Bifunctional Electrocatalysts for Hydrogen Evolution and Oxygen Evolution Reactions. ACS Sustain. Chem. Eng. 2018, 6, 12961 12968. 